\title{
Editorial
}

\section{Putting the Earth into Earth System Science}

T he International Symposium on Antarctic Earth Sciences every four years has recently been marked by active attempts to reach out to other scientific communities. The meeting in Santa Barbara in 2007 involved members of the biological community, whilst the meeting in Edinburgh in 2011 will involve scientists working on the cryosphere. Antarctica is clearly recognized, by scientists working from the outer atmosphere to the lithosphere, as a key component of the Earth System. Forming the core of the Gondwana supercontinent, its resulting polar position meant that it was the nucleation point for global glaciation from the Oligocene onwards and the records of atmospheric composition preserved in its accumulated snow and ice have given us unequalled details of our planet's climate back 750000 years.

The interdisciplinarity of Antarctic research makes it a good candidate to be the poster child of Earth System Science, a relatively new discipline that seeks to link the interactions between continents, oceans, atmosphere, ice and life using complex computer models. However, the main focus of Earth System Science is on oceans and atmospheres with only limited incorporation of living systems and human impacts and, despite its name, it does not treat the solid Earth in any meaningful way. Part of the problem is the duration of computer model runs. Few extend to more than a few centuries of elapsed time, and for global circulation model runs, a thousand year simulation can take up to six months on the fastest machines. From the point of view of the modellers, these timescales are just too short to make it worthwhile to take geological processes into account. Although increasing computer power might seem to be the obvious solution, it will not bring geological timescales into reach. As has been demonstrated many times in the past, any increase in computing capacity generates an equivalent over-demand: increased processing power is likely to be soaked up by higher model resolution and the incorporation of complex features such as clouds, or energy transfer between the ocean and atmosphere. The longest model runs always seem to converge on about six month's duration in the real world and about a thousand years of elapsed time. Rather than more computing horsepower, what is needed is a change in mindset.

Over the last ten years Earth Models of Intermediate Complexity (EMICs) have been developed that allow for much longer model runs, with the most recent models extending to tens of thousands of years. At these timescales geological processes start to become important, particularly weathering fluxes, long timescale ocean buffering by dissolution of geological formations, and the additions and losses from the atmosphere and ocean systems as a result of magmatism and metamorphism. We need to incorporate these, if, for example, we are to understand the longerterm effects and time evolution of elevated $\mathrm{CO}_{2}$ levels in the atmosphere and in the oceans. There are some glimmers of hope. A workshop in Potsdam in January 2011 showed how these models could shed light on the mechanism of past climates and carbon dioxide. The ANTScape initiative of SCAR has set itself the objectives of creating accurate palaeotopographies for Antarctica over the last 70 million years. The aim is to incorporate these into global circulation models for particular time slices, providing a new perspective for the IPCC reviews at a relatively low cost. We need more of this international and interdisciplinary leadership to drive development of sophisticated EMICs now, allowing adequate incorporation of geological processes and enabling us to move beyond simple box models for whole Earth evolution from the core to the ionosphere.

Alan P.M. Vaughan 